\title{
The Dirac spectrum in Complex Langevin Simulations of QCD
}

\author{
K. Splittorff \\ Discovery Center, The Niels Bohr Institute, University of Copenhagen, \\ Blegdamsvej 17, DK-2100, Copenhagen Ø, Denmark
}

(Dated: October 17, 2018)

\begin{abstract}
We show that the spectrum of the Dirac operator in complex Langevin simulations of QCD at nonzero chemical potential must behave in a way which is radically different from the one in simulations with ordinary non-complexified gauge fields: At low temperatures the small eigenvalues of the Dirac operator must be inside the quark mass for chemical potentials as large as a third of the nucleon mass. In particular, in the chiral limit the Dirac eigenvalues of complex Langevin simulations must accumulate at the origin.
\end{abstract}

\section{INTRODUCTION}

Complex Langevin dynamics [1, 2] is the most promising and challenging approach to the sign problem in QCD at non-zero chemical potential. It is promising because it can solve sign problems that are exponentially hard in the volume 3, 4]. It is challenging because such a success is not guaranteed [5 77. See [8] for a review.

Before we get into the details, let us recall why a first principles non-perturbative computation is necessary in order to determine the QCD phase diagram. The phase structures of strongly interacting matter are intimately linked to the transition between the hardronic phase and phases where quark and gluons are the natural degrees of freedom. The values of the chemical potential and temperatures at the transition are therefore on the hadronic scale for which QCD is highly non-perturbative. Hence the natural tool to apply is lattice QCD [9]. For zero chemical potential the fermion determinant is real and lattice QCD with two mass degenerate flavors is effectively studied using Monte Carlo sampling. One important message to take from these simulations is that the transition from hadronic matter is highly sensitive to the quark mass. A first principle non-perturbative dynamical computation is therefore essential.

At non-zero chemical potential it is natural to use complex Langevin dynamics in lattice QCD since the fermion determinant is complex. In this approach the gauge fields are complexified and one uses the complex valued action to define the drift of the gauge field configurations with Langevin time. Observables are evaluated on the trajectory traced out by the gauge fields and the expectation value is simply the average for sufficiently many Langevin time steps.

During the past year improved numerical methods $[10$ ] have allowed to simulate full QCD at non-zero chemical potential, $\mu$, with complex Langevin in different parameter regions [11, 12]. The key challenge at present is to understand if complex Langevin also allows us to simulate QCD at low temperature, small quark mass and chemical potential up to a third of the nucleon mass. This region is the test ground for numerical efforts to solve the QCD sign problem. From Monte Carlo simulations at $\mu=0$ we know that chiral symmetry is spontaneously broken, and hence the vacuum is dominated by pions. As pions are neutral wrt. the quark charge no phase transitions are expected in the region $\mu \sim m_{\pi} / 2$. However, once the chemical potential passes half the pion mass the sign problem becomes extremely severe, see e.g. [13], and unless the sign problem is under full control one is likely to see an unphysical phase transition at $\mu=m_{\pi} / 2$, see eg. [14].

The sign problem encountered beyond $\mu=m_{\pi} / 2$ becomes particularly clear when considered from the perspective of the Dirac eigenvalues. In standard QCD without any complexification of the gauge fields the eigenvalues of the Dirac operator form a band along the imaginary axis $14-16]$. When the chemical potential increases to $m_{\pi} / 2$ this band reaches the quark mass. For larger values of the chemical potential the eigenvalue density moves beyond the quark mass. In a theory without a sign problem, such as phase quenched QCD, this will imply that the theory is in a new phase characterised by pion condensation [17 19]. For full QCD, however, in a region extending from the quark mass and outward the eigenvalue density becomes complex valued and highly oscillatory [16]. See Fig. 1 and 2 upper panels. These oscillations wipe out the unphysical phase transition at $\mu=m_{\pi} / 2[20,21]$ and cause the discontinuity of the chiral condensate at zero quark mass.

In this paper we consider, for the first time, the general properties of the eigenvalues of the Dirac operator evaluated on the complexified gauge field configurations generated by the complex Langevin dynamics. We show that the behaviour of the Dirac spectrum in complex Langevin simulations must be drastically different from that with real gauge fields: The real part of the small eigenvalues of the Dirac operator in complex Langevin must have a magnitude less than the quark mass. This holds for low temperature and chemical potentials as large as a third of the nucleon mass. In particular, in the chiral limit the small Dirac eigenvalues of complex Langevin simulations must accumulate at the origin. See the lower panels of Fig. 1 and 2. The accumulation of Dirac eigenvalues at the origin is in sharp contrast to the fact that the chemical potential breaks the anti-Hermiticity of the Dirac 
operator but, as we will show, it is not excluded by the standard arguments.

To set the stage we first recall the two different links between the spectrum of the Dirac operator with real gauge fields and the chiral condensate at zero [22] and non-zero chemical potential [20, 21]. We then turn to the spectrum of the complex Langevin Dirac operator at non-zero $\mu$. The 7 main points are highlighted by enumeration.

\section{BANKS-CASHER AT $\mu=0$}

The successful application of importance sampling in lattice QCD for 2 degenerate quark flavors at $\mu=0$ relies on the fact that we (in this case) deal with a statistical system: The fermion determinant is real and its square positive. One way to see this is to express the determinant in terms of the eigenvalues $i \lambda_{k}$ of the Dirac operator at $\mu=0$

$$
\begin{aligned}
\operatorname{det}\left(D_{\mu=0}+m\right) & =\prod_{k}\left(i \lambda_{k}+m\right)\left(-i \lambda_{k}+m\right) \\
& =\prod_{k}\left(\lambda_{k}^{2}+m^{2}\right),
\end{aligned}
$$

where we have used that the Dirac operator is antiHermitian and anti-commutes with $\gamma_{5}$. For simplicity we do not consider topological zero modes.

A central observable which gives us insights on the phases of strongly interacting matter is the chiral condensate,

$$
\Sigma(m)=\left\langle\operatorname{Tr} \frac{1}{D_{\mu=0}+m}\right\rangle_{N_{f}, \mu=0} .
$$

Here $\langle\ldots\rangle_{N_{f}, \mu=0}$ denotes the QCD expectation value with $N_{f}$ dynamical quark flavors of equal mass $m$ and zero chemical potential $\mu$. Expressed in terms of the Dirac eigenvalues we have

$$
\Sigma(m)=\left\langle\sum_{k} \frac{1}{i \lambda_{k}+m}\right\rangle_{N_{f}, \mu=0} .
$$

Equivalently, we can write this as (for a detailed discussion see [23])

$$
\Sigma(m)=\int d \lambda \rho_{N_{f}}(\lambda, m) \frac{1}{i \lambda+m}
$$

where the eigenvalue density is defined by

$$
\rho_{N_{f}}(\lambda, m)=\left\langle\sum_{k} \delta\left(\lambda-\lambda_{k}\right)\right\rangle_{N_{f}, \mu=0} .
$$

Since the non-zero eigenvalues come in pairs of opposite sign on the imaginary axis the integrand of (4) forms a $\delta$-function in the chiral limit, $m \rightarrow 0$, which singles out $\lambda=0$. This gives the Banks-Casher relation 22]

$$
\lim _{m \rightarrow 0} \Sigma(m)=\frac{\pi}{V} \rho(0) .
$$

In words: at zero chemical potential the density of Dirac eigenvalues at the origin of the imaginary axis is proportional to the chiral condensate in the chiral limit.

\section{NON-COMPLEXIFIED GAUGE FIELDS AT $\mu \neq 0$}

Next, let us consider QCD at non-zero chemical potential, without any complexification of the gauge fields. The first thing to notice is that the standard BanksCasher relation (6) is invalidated. Since the Dirac operator is no longer anti-Hermitian the eigenvalues, $z_{k}$, of the Dirac operator, $D_{\mu}$, move into the complex plane. The chiral condensate,

$$
\Sigma(m ; \mu)=\left\langle\operatorname{Tr} \frac{1}{D_{\mu}+m}\right\rangle_{N_{f}, \mu},
$$

can still be expressed in terms of the eigenvalues $z_{k}$ of $D_{\mu}$

$$
\begin{aligned}
\Sigma(m ; \mu) & =\left\langle\sum_{k} \frac{1}{z_{k}+m}\right\rangle_{N_{f}, \mu} \\
& =\int d^{2} z \rho_{N_{f}}(z, m ; \mu) \frac{1}{z+m}
\end{aligned}
$$

where the eigenvalue density is

$$
\rho_{N_{f}}(z, m ; \mu)=\left\langle\sum_{k} \delta^{(2)}\left(z-z_{k}\right)\right\rangle_{N_{f}, \mu} .
$$

However, eventhough the non-zero eigenvalues still come in pairs of opposite sign, the integrand of (9) does not form a $\delta$-function at the origin of the complex plane for $m \rightarrow 0$. The standard Banks-Casher relation (6) is therefore not valid at non-zero $\mu$.

What replaces the Banks-Casher relation is the fact that

1) $\rho_{N_{f}}(z, m ; \mu)$ will take complex values for $\mu>m_{\pi} / 2$ [16].

2) These complex valued oscillations of $\rho_{N_{f}}(z, m ; \mu)$ are responsible for the formation of the chiral condensate [20, 21].

For illustrations, see the upper panels of Figure 1 and Figure 2] as well as 24].

It was possible to obtain these insights since the low lying eigenvalue density can be determined uniquely from the flavor symmetries through chiral random matrix theory [25] and chiral perturbation theory [16]. The fact that 

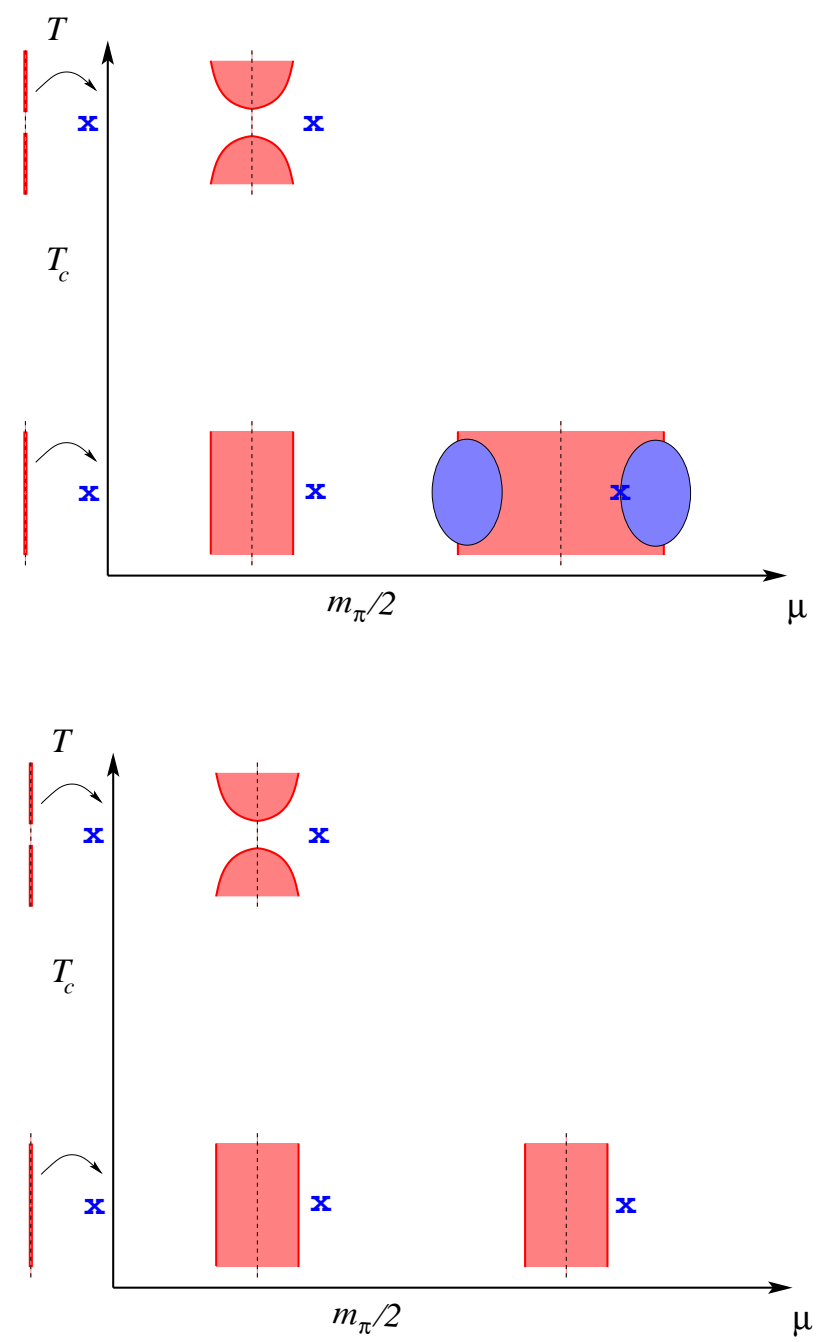

FIG. 1: Sketch of the low lying Dirac spectrum various places in the $(\mu, T)$-plane. The quark mass, illustrated by the blue cross, is fixed. Top: For ordinary real gauge fields, Below: in complex Langevin simulations. Within the red region the eigenvalue density is real and positive. The region where the eigenvalue density is complex valued and rapidly oscillating is marked in blue. This region is not possible to realise for complex Langevin, hence the eigenvalues must necessarily stay inside the quark mass even if $\mu>m_{\pi} / 2$.

the eigenvalue density takes complex values for $\mu>m_{\pi} / 2$ is possible because the $\delta$-function in (9) is weighted by the complex valued fermion determinant. The contribution from this oscillating part of the eigenvalue density to the integral in (9) increases as the quark mass approaches zero and it changes sign as the quark mass passes through the origin. Hence, the complex and oscillating part of the spectral density is responsible for the spontaneous breaking of chiral symmetry [20,21]. Moreover, the oscillations ensures that $\Sigma(m ; \mu)$ is smooth at $\mu=m_{\pi} / 2$.
Phase quenched QCD

In contrast, for phase quenched theory $\mathrm{QCD}$, where the fermion determinant is replaced by its absolute value, there is no sign problem and the eigenvalue density is necessarily real and positive. It forms a band along the imaginary axis of width $2 \mu^{2} F_{\pi}^{2} / \Sigma$, independent of the quark mass [15, 19]. From this we learn that

3) a positive density of eigenvalues in a band along the imaginary axis, leads to a vanishing value of the chiral condensate in the chiral limit, for any non-zero value of $\mu$.

4) a phase transition occurs when the quark mass hits this positive eigenvalue density.

The quark mass hits the strip of eigenvalues at $\mu=m_{\pi} / 2$ and the new phase for $\mu>m_{\pi} / 2$ is characterized by a non-zero pion condensate [17] [41].

\section{COMPLEXIFIED GAUGE FIELDS AT $\mu \neq 0$}

Now let us turn to the complex Langevin approach. Given an action $S(\mathbf{x})$ which takes complex values when evaluated on the real fields $\mathbf{x}=\left(x_{1}, \ldots, x_{N}\right)$ the standard form of the complex Langevin drift equations, obtained by complexifying all fields $x_{k} \rightarrow x_{k}+i y_{k}$, is [1, 2, [8]

$$
\begin{aligned}
& x_{k}^{(t+1)}=x_{k}^{(t)}-\operatorname{Re}\left[\frac{d S}{d x_{k}}\right]_{\mathbf{x}=\mathbf{x}^{(\mathbf{t})}+\mathbf{i} \mathbf{y}^{(\mathbf{t})}} d t+\eta \sqrt{d t} \\
& y_{k}^{(t+1)}=y_{k}^{(t)}-\operatorname{Im}\left[\frac{d S}{d x_{k}}\right]_{\mathbf{x}=\mathbf{x}^{(\mathbf{t})}+\mathbf{i} \mathbf{y}^{(\mathbf{t})}} d t .
\end{aligned}
$$

Here $\eta$ is the Langevin noise term, see e.g. [8]. The suberscript $t=1, \ldots, T$ refers to the Langevin time and expectation values of an observable $\mathcal{O}(\mathbf{x})$ are measured simply by averaging over the observable evaluated on the Langevin trajectory for sufficiently large $T$

$$
\langle\mathcal{O}\rangle=\frac{1}{T} \sum_{t=1}^{T} \mathcal{O}\left(x_{k}^{(t)}+i y_{k}^{(t)}\right) .
$$

In applications to $\mathrm{QCD}$ at $\mu \neq 0$ the fermions are integrated out and a trajectory of complexified gauge field configurations, $A_{\nu}^{(t)}$, is obtained from the complex Langevin drift equations [1].

The Dirac eigenvalues, $z_{k}^{(t)}$, on the Langevin trajectory of complexified gauge fields, $A_{\nu}^{(t)}$, are determined through the eigenvalue equation

$$
D_{\mu}\left(A_{\nu}^{(t)}\right) \psi_{k}=z_{k}^{(t)} \psi_{k}
$$

The general properties of the eigenvalues, $z_{k}^{(t)}$, are much the same as with real gauge fields and non-zero chemical 
potential: The anti-commutation of the Dirac operator, $D_{\mu}\left(A_{\nu}^{(t)}\right)$, with $\gamma_{5}$ is conserved but the anti-Hermiticity is broken. The non-zero eigenvalues, $z_{k}^{(t)}$, therefore come in pairs of opposite sign in the complex plane.

A natural question to ask at this point is: can complex Langevin realize the formation of the chiral condensate through the oscillating eigenvalue density as in the ordinary approach to $Q C D$. The answer is: No.

To see why, let us now consider the formation of the chiral condensate in complex Langevin simulations of QCD. The Langevin process will generate a trajectory with complexified gauge field configurations, $A_{\nu}^{(t)}$, at Langevin time $t$ and the chiral condensate measured is

$$
\Sigma^{(C L)}(m)=\frac{1}{T} \sum_{t} \operatorname{Tr} \frac{1}{D_{\mu}\left(A_{\nu}^{(t)}\right)+m},
$$

Expressed in terms of the Dirac eigenvalues we have

$$
\Sigma^{(C L)}(m)=\frac{1}{T} \sum_{t} \sum_{k} \frac{1}{z_{k}^{(t)}+m} .
$$

Analogously to the standard case above let us rewrite this as

$$
\Sigma(m)=\int d^{2} z \frac{\rho_{N_{f}}^{(C L)}(z, m ; \mu)}{z+m},
$$

where

$$
\rho_{N_{f}}^{(C L)}(z, m ; \mu)=\frac{1}{T} \sum_{t} \sum_{k} \delta^{(2)}\left(z-z_{k}^{(t)}\right) .
$$

While this at first appears to be similar to the density for real gauge fields, (9), there is one key difference

5) $\rho_{N_{f}}^{(C L)}(z, m ; \mu)$ is by definition real and positive.

Therefore, it is not possible to realise the formation of the chiral condensate through extreme and complex valued oscillations of the eigenvalue density.

A next natural question is therefore: Does this imply that the complex Langevin approach fails for $\mu>m_{\pi} / 2$ ? The answer is: No, not nessesarely.

Eventhough $\rho_{N_{f}}^{(C L)}(z, m ; \mu)$ is positive, the unphysical phase transition is avoided provided that

6) the density of the small eigenvalues must be located within a band bounded by $\left|\operatorname{Re}\left[z_{k}^{(t)}\right]\right|<|m|$ in the vicinity of the real axis.

If not there will be a phase transition at the point where the quark mass hits the boundary of the eigenvalues. In particular,
7) in the chiral limit the small eigenvalues of the Dirac operator evaluated on the complexified configurations must accumulate to a diverging density at the origin.

In other words: Despite the fact that the chemical potential breaks the anti-Hermiticity of the Dirac operator, the small eigenvalues of the Dirac operator must be pushed to the origin by the decreasing value of the quark mass. This is exemplified in the appendix.

The next question is to ask is, of course, is this possible?

A natural way to get eigenvalues with small real part is from an anti-Hermitian operator. However, since the gauge group of QCD is $\mathrm{SU}(3)$, it is not possible to choose a complexified gauge field configuration for which the Dirac operator at non-zero chemical potential is antiHermitian. To see this explicitly let us write the Hermitian and anti-Hermitian part of the complexified gauge fields, $A_{\nu}^{(t)}$ as $A_{\nu}^{(t)}=X_{\nu}^{(t)}+i Y_{\nu}^{(t)}$, where $X_{\nu}^{(t)}$ and $Y_{\nu}^{(t)}$ are Hermitian. We then have

$$
\begin{aligned}
D\left(A_{\nu}^{(t)}\right)^{\dagger} & =\left(\gamma_{\mu}\left(\partial_{\nu}+i\left(X_{\nu}^{(t), a}+i Y_{\nu}^{(t), a}\right) \lambda^{a}+\mu \delta_{\nu, 0}\right)\right)^{\dagger} \\
& =-\gamma_{\mu}\left(\partial_{\nu}+i\left(X_{\nu}^{(t), a}-i Y_{\nu}^{(t), a}\right) \lambda^{a}-\mu \delta_{\nu, 0}\right)
\end{aligned}
$$

where the $\lambda^{a}$ are the color generators of SU(3). To make the full Dirac operator anti-Hermitian would require that the $Y_{\nu}^{(t), a}$ nulify the chemical potential and this is not possible since the unit matrix in color is not a generator of SU(3). A total anti-Hermitization of the Dirac operator by complixification of the gauge fields is therefore not possible [42]. The new constraints on the spectrum found above, however, only require that the small Dirac eigenvalues accumulate at the origin in the chiral limit. For this a total anti-Hermitization of the Dirac operator is not required.

The fact that the quark mass hits the spectrum of the Dirac operator at $\mu=m_{\pi} / 2$ for real gauge fields is not accidental: This follows directly from the spontaneous breakdown of chiral symmetry and the fact that the complex conjugate of the fermion determinant equals the determinant with the opposite sign of the chemical potential [15, 19, 26]. This standard argument does not exclude the possibility that the spectral density of the Dirac operator with complexified gauge fields remains inside the quark mass even if $\mu>m_{\pi} / 2$ : In the case of complex Langevin the complex conjugate of the fermion determinant is

$$
\begin{gathered}
\operatorname{det}\left(\gamma_{\mu}\left(\partial_{\nu}+i\left(X_{\nu}^{(t), a}+i Y_{\nu}^{(t), a}\right) \lambda^{a}+\mu \delta_{\nu, 0}\right)+m\right)^{*} \\
=\operatorname{det}\left(\gamma_{\mu}\left(\partial_{\nu}+i\left(X_{\nu}^{(t), a}-i Y_{\nu}^{(t), a}\right) \lambda^{a}-\mu \delta_{\nu, 0}\right)+m\right) .
\end{gathered}
$$

For non-zero values of $Y_{\nu}^{(t)}$ (i.e. when the gauge field is complex) this is not equal to the determinant at $-\mu$. Hence spontaneous breaking of chiral symmetry does not 


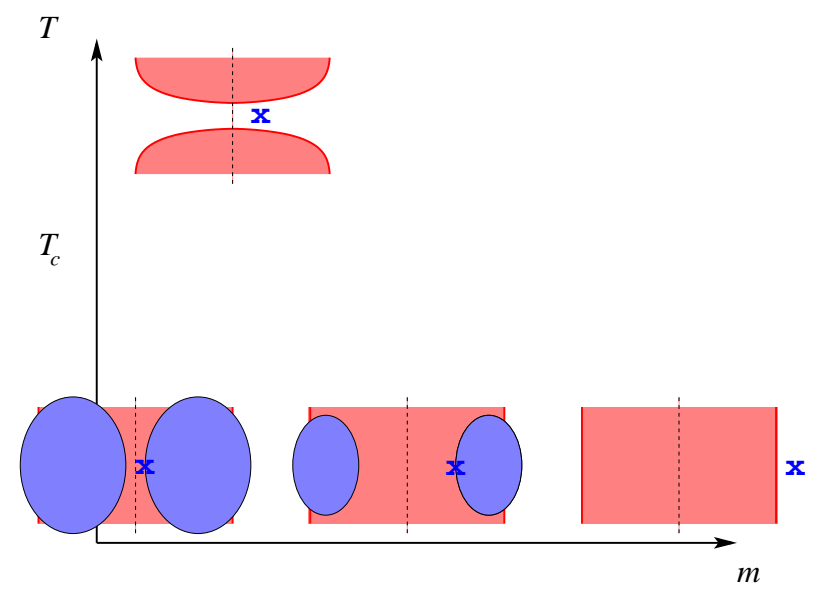

$T$

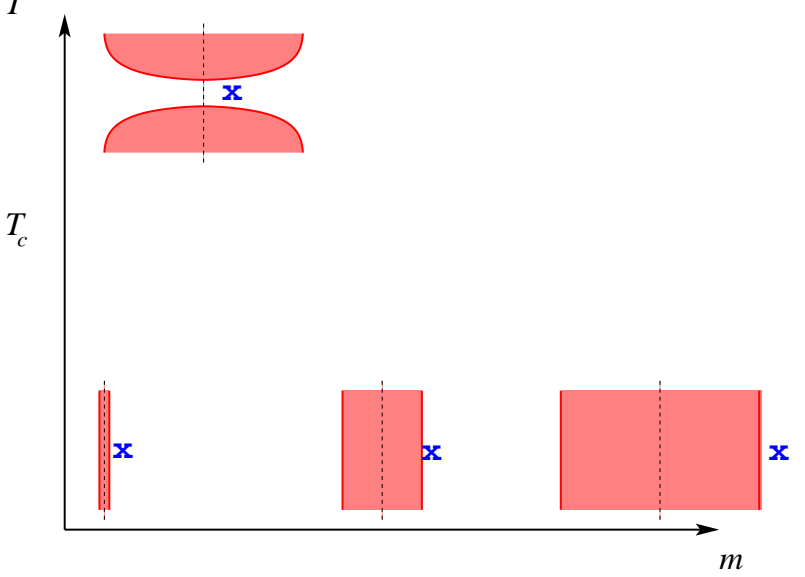

FIG. 2: The schematic behaviour of the low lying Dirac spectrum in the $(m, T)$-plane at fixed non-zero $\mu$ (where $|\mu|$ is less than a third of the nucleon mass). Top: with real gauge fields, Below: with complexified fields from complex Langevin. The eigenvalue density is real and positive within the red region and complex within the blue. For real gauge fields the quark mass (marked by the blue $\mathrm{X}$ ) hits the eigenvalue density when $m_{\pi}=2 \mu$. In complex Langevin simulations the eigenvalues must be inside the quark mass.

automatically imply that the quark mass hits the support of the Dirac spectrum with complexified gauge fields for $\mu=m_{\pi} / 2$. The necessary behaviour of the small Dirac eigenvalues in complex Langevin simulations sketched in the lower panels of Figure 1 and 2 and exemplified in the appendix, is therefore not excluded by the standard argument.
The cut of the log and a practical suggestion for simulations

As demonstrated in [7] the presence of the log of the fermion determinant in the action may lead to inconsistencies if the determinant frequently circles the origin during the Langevin flow, see also 27]. These inconsistencies arise because the complex Langevin drift includes the derivative of the non-holomorfic logarithm in the complex plane. This becomes particularly relevant when the argument of the log passes the cut of the log.

If we express the $\log$ of the fermion determinant through the eigenvalues of the Dirac operator,

$$
\log \operatorname{det}\left(D_{\mu}\left(A_{\nu}^{(t)}\right)+m\right)=\sum_{k} \log \left(z_{k}^{(t)}+m\right),
$$

the potential problems with the log become relevant when the quark mass is inside the support of the eigenvalues, such that $z_{k}^{(t)}+m$ or $-z_{k}^{(t)}+m$ can circle the origin of the complex plane (recall that for every non-zero $z_{k}^{(t)}$ there is an eigenvalue $\left.-z_{k}^{(t)}\right)$. Now, as we have seen above, in order that complex Langevin correctly describes the spontaneous breaking of chiral symmetry, at low temperature and $\mu$ up to a third of the nucleon mass, the small eigenvalues must end up inside the quark mass. If the eigenvalues indeed do so the problems with the log will be minimal and complex Langevin can be a solution to the QCD sign problem.

If the complex Langevin eigenvalues end up inside the quark mass there still remains, a problem of 'thermalization': If at Langevin time, $t=1$, the trajectory starts from a typical (i.e. a quenched, a $\mu=0$, or a phase quenched) real gauge field configuration the quark mass will initially be inside the support of the Dirac eigenvalues if $\mu>m_{\pi} / 2$. Therefore with Langevin time the eigenvalues must must flow inside the quark mass. However, in order to safely ignore the issues with the log, the Langevin flow of the Dirac eigenvalues must be such that the eigenvalues do not circle the quark mass. Even if the Langevin drift may not wish to drive the eigenvalues around the quark mass [43] it will take a very careful update procedure (a small time step) to avoid that this happens due to the Langevin noise: If the quark mass initially is inside the support of the Dirac eigenvalues then the typical distance from the quark mass to the nearest eigenvalue is of order the inverse volume [16] and a numerical fluctuation can cause this eigenvalue to move to the other side of the quark mass. In practice, it is also exceedingly demanding to invert the Dirac operator numerically when the quark mass is inside the cloud of eigenvalues.

In order to overcome this we here suggest initially to let the quark mass depend on the Langevin time. If the Langevin trajectory starts from a typical real gauge field configuration and we pick a quark mass such that it is 
close to but outside the eigenvalues of the Dirac operator on the original configuration, then we can run the Langevin flow ignoring the issues with the cut of the log. If at a suitable Langevin time $t_{1}$ the small eigenvalues of the Dirac operator evaluated on the latest complex valued configuration is in a narrower band than at time $t=1$ we then decrease the quark mass towards the desired value but no further than it is still outside the band of Dirac eigenvalue at $t_{1}$. Continuing in this manner one may potentially take the quark mass all the way to the desired value without ever having the quark mass inside the support of the eigenvalues. Once the desired value of the quark mass is reached, all prior configurations are discarded in the Langevin measurement. Alternatively one may work at a fixed quark mass and increase the chemical potential with Langevin time until the desired value is reached [44].

\section{CONCLUSIONS}

We have considered, for the first time, the general properties of the Dirac eigenvalues on the complexified gauge field configurations generated by complex Langevin. By the very definition of the expectation value in complex Langevin the density of Dirac eigenvalues on the Langevin trajectory of complexified gauge fields will be real and positive. Therefore, using complex Langevin simulations it is not possible to realize the complex and oscillating eigenvalues density which is essential for the spontaneous breaking of chiral symmetry with real gauge fields. The eigenvalues on the complexified gauge fields must therefore distribute themselves in a manner which is drastically different from the distribution obtained with real gauge fields: The small Dirac eigenvalues, when evaluated on the Langevin trajectory, must form a band inside the quark mass. In particular, in the chiral limit the small eigenvalues must accumulate at the origin.

A non-positive Dirac eigenvalue density where oscillations with a period of order the inverse volume and an exponentially large amplitude is a generic feature of theories with a fermionic sign problem. It is essential in the microscopic domain of QCD [20, 21, 28], for Cooper paring at large chemical potential 29], in one dimensional QED at any chemical potential [4, 30], in two color QCD with unmatched quark masses [31], as well as for odd flavored QCD at zero chemical potential and a negative quark mass [32]. All cases are characterized by a fermonic sign problem and the results obtained here also applies in these cases: If complex Langevin is applied the resulting Dirac spectrum must be drastically different from the one obtained with real fields.

Finally, we have proposed to work with an initially Langevin time dependent quark mass. It would be most interesting to implement this in complex Langevin simulations of full QCD. A first test in the context of chiral random matrix theory [25, 33] is presented in [34].

Acknowledgments: Jac Verbaarschot, Poul Henrik Damgaard, Mario Kieburg, Gernot Akemann, Philippe de Forcrand, Frithjof Karsch, Gert Aarts, Jeff Greensite, Francesco Di Renzo, Erhard Seiler, Christian Schmidt, Denes Sexty, Nucu Stamatescu, Luigi Scorzato, Joyce C. Myers and Anders Mollgaard as well as participants of Sign 2014, XQCD 2014 and 'Conceptual advances in lattice gauge theory (LTG14 at CERN)' are thanked for discussions. The work of KS was supported by the Sapere Aude program of The Danish Council for Independent Research.

\section{THREE EXAMPLES}

We here give three examples of positive spectral densities and discuss the resulting chiral condensates.

\section{1) a uniform strip of width $2 \mu^{2} F_{\pi}^{2} / \Sigma$}

The first example is the positive eigenvalue density obtained in phase quenched QCD. At mean field level in chiral perturbation theory [15, 35] it forms a strip around the imaginary axis of width $2 \mu^{2} F_{\pi}^{2} / \Sigma$. Within this strip the density is constant

$$
\rho(z, m ; \mu)=\frac{\Sigma^{2}}{4 \pi \mu^{2} F_{\pi}^{2}} \theta\left(2 \mu^{2} F_{\pi}^{2} / \Sigma-|x|\right),
$$

where $z=x+i y$. Note that the eigenvalue density is independent of the quark mass and that the normalization of the density requires that the density itself diverges as $\mu \rightarrow 0$.

The resulting chiral condensate,

$$
\begin{aligned}
\Sigma(m ; \mu)= & \frac{\Sigma^{2}}{2 \mu^{2} F_{\pi}^{2}} m \theta\left(2 \mu^{2} F_{\pi}^{2} / \Sigma-|m|\right) \\
& +\Sigma \operatorname{sign}(m) \theta\left(-2 \mu^{2} F_{\pi}^{2} / \Sigma+|m|\right),
\end{aligned}
$$

has no discontinuity as $m$ passes through zero. Moreover, the kink at $m=2 \mu^{2} F_{\pi}^{2} / \Sigma$ is the signal of the second order phase transition at $\mu=m_{\pi} / 2$ as follows using the GOR relation $m_{\pi}^{2} F_{\pi}^{2}=2 \Sigma m$.

\section{2) a uniform strip of width $|m| / 2$}

In full QCD with real gauge fields the eigenvalue density is complex and depends heavily on the quark mass $m$. The Dirac eigenvalue density in complex Langevin simulations of full QCD is real and positive definite by construction and the support must be inside the quark mass for low temperature. To exemplify this we consider here an example of a positive eigenvalue density which is in a strip of width $|m| / 2$

$$
\rho(z, m ; \mu)=\frac{\Sigma}{\pi|m|} \theta(|m| / 2-|x|),
$$


where $x=\operatorname{Re}[z]$. In order to make the example more realistic let us assume that this is true for $|m|<4 \mu^{2} F_{\pi}^{2} / \Sigma$ while for larger values of $|m|$ the density is given as in the first example above.

Note that this eigenvalue density diverges as $m \rightarrow 0$ in order to keep the normalization fixed (it becomes a onedimensional density on the imaginery axis for $m=0$ ). This leads to a discontinuous chiral condensate,

$$
\Sigma(m)=\Sigma \operatorname{sign}(m)
$$

signalling the spontaneous breakdown of chiral symmetry in the chiral limit.

The diverging density at the origin in the chiral limit exemplifies the behaviour which the eigenvalue density must have in complex Langevin simulations in order to support the spontaneous breaking of chiral symmetry.

\section{3) scaling relation}

It is tempting to come up with examples where the eigenvalue density for a real number $k$ satisfies the scaling relation

$$
\rho(k z, k m ; \mu)=\frac{1}{|k|} \rho(z, m ; \mu) .
$$

For example

$$
\rho(z, m ; \mu)=\frac{\Sigma}{2|y|+|m|} \frac{1}{\pi} \theta(|y|+|m / 2|-|x|),
$$

where $z=x+i y$ so that the support has the form of an hourglass. The minimal width $|m| / 2$ is obtained at $y=0$ and from there the width grows strictly linearly with $|y|$.

With the scaling relation (23) we automatically get that

$$
\begin{aligned}
\Sigma(k m) & =\int d x d y \frac{1}{x+i y+k m} \rho(z, k m ; \mu) \\
& =k^{2} \int d x^{\prime} d y^{\prime} \frac{1}{k x^{\prime}+i k y^{\prime}+k m} \rho\left(k z^{\prime}, k m ; \mu\right) \\
& =\frac{k^{2}}{k|k|} \int d x^{\prime} d y^{\prime} \frac{1}{x^{\prime}+i y^{\prime}+m} \rho\left(z^{\prime}, m ; \mu\right) \\
& =\operatorname{sign}(k) \Sigma(m),
\end{aligned}
$$

where $z=x+i y$ and $z=k z^{\prime}$. As desired the condensate is $m$ independent except that it changes sign at $m=0$. There is a catch, however, since the scaling relation (23) is inconsistent with the fact that the normalization of the eigenvalue density,

$$
\int d^{2} z \rho(z, m ; \mu)
$$

must be independent of $m$. For this to hold true the value of $k$ in (23) must be 1 and the scaling relation is therefore trivial.

[1] G. Parisi, Phys. Lett. B 131, 393 (1983).
[2] J. R. Klauder, J. Phys. A 16, L317 (1983).

[3] G. Aarts, Phys. Rev. Lett. 102, 131601 (2009) arXiv:0810.2089 [hep-lat]].

[4] G. Aarts and K. Splittorff, JHEP 1008, 017 (2010) arXiv:1006.0332 [hep-lat]].

[5] J. Ambjorn, M. Flensburg and C. Peterson, Nucl. Phys. B 275, 375 (1986).

[6] G. Aarts and F. A. James, JHEP 1008, 020 (2010) arXiv:1005.3468 [hep-lat]].

[7] A. Mollgaard and K. Splittorff, Phys. Rev. D 88, 116007 (2013) arXiv:1309.4335 [hep-lat]].

[8] G. Aarts, PoS LATTICE 2012, 017 (2012) arXiv:1302.3028 [hep-lat]].

[9] P. de Forcrand, PoS LAT 2009, $010 \quad$ (2009) arXiv:1005.0539 [hep-laxt]].

[10] E. Seiler, D. Sexty and I. O. Stamatescu, Phys. Lett. B 723, 213 (2013) arXiv:1211.3709 [hep-lat]].

[11] D. Sexty, Phys. Lett. B 729, 108 (2014) arXiv:1307.7748 [hep-lat]].

[12] G. Aarts, E. Seiler, D. Sexty and I. O. Stamatescu, arXiv:1408.3770 [hep-lat].

[13] K. Splittorff and J. J. M. Verbaarschot, Phys. Rev. Lett. 98, 031601 (2007) hep-lat/0609076 ; Phys. Rev. D 75, 116003 (2007) hep-lat/0702011 [HEP-LAT]].

[14] K. Splittorff, PoS LAT 2006, 023 (2006) hep-lat/0610072.

[15] D. Toublan and J. J. M. Verbaarschot, Int. J. Mod. Phys. B 15, 1404 (2001) hep-th/0001110.

[16] G. Akemann, J. C. Osborn, K. Splittorff and J. J. M. Verbaarschot, Nucl. Phys. B 712, 287 (2005) hep-th/0411030.

[17] D. T. Son and M. A. Stephanov, Phys. Rev. Lett. 86, 592 (2001) hep-ph/0005225.

[18] K. Splittorff, D. T. Son and M. A. Stephanov, Phys. Rev. D 64, 016003 (2001) hep-ph/0012274.

[19] J. C. Osborn, K. Splittorff and J. J. M. Verbaarschot, Phys. Rev. D 78, 105006 (2008) arXiv:0807.4584 [heplat]].

[20] J. C. Osborn, K. Splittorff and J. J. M. Verbaarschot, Phys. Rev. Lett. 94, 202001 (2005) hep-th/0501210.

[21] J. C. Osborn, K. Splittorff and J. J. M. Verbaarschot, Phys. Rev. D 78, 065029 (2008) arXiv:0805.1303 [hepth]].

[22] T. Banks and A. Casher, Nucl. Phys. B 169, 103 (1980).

[23] H. Leutwyler and A. V. Smilga, Phys. Rev. D 46, 5607 (1992).

[24] J. C. Osborn, K. Splittorff and J. J. M. Verbaarschot, hep-lat/0510118

[25] J. C. Osborn, Phys. Rev. Lett. 93, 222001 (2004) hep-th/0403131.

[26] M. A. Stephanov, Phys. Rev. Lett. 76, 4472 (1996) hep-lat/9604003.

[27] J. Greensite, arXiv:1406.4558 [hep-lat].

[28] J. R. Ipsen and K. Splittorff, Phys. Rev. D 86, 014508 (2012) arXiv:1205.3093 [hep-lat]].

[29] T. Kanazawa and T. Wettig, arXiv:1406.6131 [hep-ph].

[30] L. Ravagli and J. J. M. Verbaarschot, Phys. Rev. D 76, 054506 (2007) arXiv:0704.1111 [hep-th]].

[31] G. Akemann, T. Kanazawa, M. J. Phillips and T. Wettig, JHEP 1103, 066 (2011) arXiv:1012.4461 [hep-lat]].

[32] J. J. M. Verbaarschot and T. Wettig, arXiv:1407.8393 [hep-th].

[33] J. Bloch, F. Bruckmann, M. Kieburg, K. Splittorff and J. J. M. Verbaarschot, Phys. Rev. D 87, no. 3, 034510 
(2013) arXiv:1211.3990 [hep-lat]].

[34] A. Mollgaard and K. Splittorff, in preparation.

[35] K. Splittorff and J. J. M. Verbaarschot, Nucl. Phys. B 683, 467 (2004) hep-th/0310271.

[36] F. Karsch, Nucl. Phys. A 461, 305C (1987).

[37] M. Kieburg, K. Splittorff and J. J. M. Verbaarschot, Phys. Rev. D 85, 094011 (2012) arXiv:1202.0620 [heplat]].

[38] M. Cristoforetti et al. [AuroraScience Collaboration], Phys. Rev. D 86, 074506 (2012) arXiv:1205.3996 [heplat]].

[39] G. Aarts, Phys. Rev. D 88, no. 9, 094501 (2013) arXiv:1308.4811 [hep-lat]].

[40] G. Aarts, L. Bongiovanni, E. Seiler and D. Sexty, arXiv:1407.2090 [hep-lat].

[41] A real and positive eigenvalue density which forms a strip in the complex plane can lead to a discontinuity of the chiral condensate at $m=0$ if $\rho(z, m ; \mu) \neq \rho(-z, m ; \mu)$
[37].

[42] Note that with gauge group U(3) the Dirac operator at non-zero $\mu$ can be absorbed into a global shift of the gauge fields in the complex plane. Complex Langevin simulations for QCD with gauge group $\mathrm{U}(3)$ have been reported to be successful [36].

[43] Trajectories where the imaginary part of the action remain constant and which end up at fixed points are known as Lefschetz thimbles 38]. In 39, 40] it was demonstrated that in some specific examples, the regions of the complex plane sampled by complex Langevin are in the neighbourhood of the Lefschets thimble. If this is true in full QCD then the determinant does not frequently circle the origin.

[44] I thank Prof. F. Karsch for this suggestion given during a seminar at University of Bielefeld December 2013. 